\title{
Seguridad Laboral y Salud Ocupacional en los Hospitales del Ecuador
}

\section{Occupational Safety and Health in the Hospitals of Ecuador}

\section{Segurança e Saúde Ocupacional nos Hospitais do Equador}

\author{
Karen J. Cedeño-Álava I \\ karen.cedeno-@yahoo.com \\ María Elena De la Cruz Santillán II \\ maria.santillan1@gmail.com \\ María J. Zambrano-Zambrano ${ }^{\mathrm{III}}$ \\ maria-45zam@gmail.com
}

\author{
Gema J. Cantos-Alcívar IV \\ gemacantos@gmail.com \\ Stalin A. Intriago-Miranda V \\ stalin-intriago@gmail.com \\ Rina G. Soledispa-Canizares VI \\ rina.soledispa@hotmail.com
}

Recibido: 27 de junio de 2018 * Corregido: 28 de agosto de $2018 *$ Aceptado: 20 de septiembre de 2018

I. Medico Ocupacional, Coordinación zonal 4 Manabí, Santo Domingo, Ecuador.

II. Médico Tratante; Distrito de Salud 13d06 Bolívar Calceta, Ecuador.

III. Médico General; Centro de Salud a IESS Calceta, Ecuador.

IV. Médico responsable del Banco de Leche Humano Hospital Verdi Cevallos Balda, Portoviejo, Ecuador.

v. Médico en Funciones Hospitalarias, Responsable de la Gestión y Epidemiología Hospitalaria, Médico certificado en Prevención de Riesgos Laborales, Maestrante de la Maestría en Seguridad y Salud Ocupacional Universidad San Gregorio en Portoviejo, Ecuador.

VI. Licenciada en Enfermería, Enfermera responsable de Unidad de Cuidados Intensivos Pediátricos Hospital Verdi Cevallos, Portoviejo, Ecuador. 


\section{Resumen}

La presente investigación pretende analizar la importancia que tiene el cumplimento de las normas en la salud ocupacional enfocado a la prevención de accidentes y riesgos las diferentes acciones que afectan en el trabajo y la producción, el manejo de materiales, tanto químicos, físicos, o biológicos; además de algunos aspectos de seguridad. La tendencia moderna está dirigida precisamente a considerarlos como órganos de gestión, no solamente como asesores, como era la orientación en sus orígenes. Por eso mismo integran los Servicios Operativos, órganos ejecutivos de primer orden para la gestión que se necesita realizar. Los accidentes de trabajo y enfermedades profesionales están reconocidos legalmente en el Ecuador, normativas que tiene su origen en convenios y resoluciones internacionales adoptadas por este país. El tema se abordará desde varios enfoques por lo tanto es necesario converger en la aplicación de varios métodos teóricos y empíricos. La observación, el análisis y la síntesis, la inducción y la deducción y el análisis histórico-lógico, estudios comparativos, convergieron como la estrategia metodológica, para concluir en algunos principios básicos, como aporte a el tema.

Palabras claves: Salud ocupacional, Prevención, Seguridad, Accidentes. 


\section{Abstract}

This research aims to analyze the importance that has focused on occupational health standards compliance to the prevention of accidents and risks the actions affecting labour and production, material handling, both chemists, physical, or biological; In addition to some aspects of security. The modern trend is directed precisely to consider them as management bodies, not only as advisors, as it was the orientation in its origins. Why integrate services operations, executive bodies of the first order for management that needs to be done. Accidents at work and occupational diseases are recognized legally in Ecuador, regulations that has its origin in conventions and international resolutions adopted by this country. The issue will be addressed from various approaches therefore need to converge in the implementation of several theoretical and empirical methods. Observation, analysis and synthesis, induction and deduction and historical-logical analysis, comparative studies, They converged as the methodological strategy, to conclude on some basic principles, as a contribution to the theme.

Key words: Occupational health, prevention, safety, accidents. 


\section{Introducción.}

En este artículo se pretende dar una revisión y un aporte sobre el tema de la seguridad y salud ocupacional en los hospitales en Ecuador

Se puede definir como riesgo laboral como “... el conjunto de factores físicos, psíquicos, químicos, ambientales, sociales y culturales que actúan sobre el individuo; la interrelación y los efectos que producen esos factores dan lugar a la enfermedad ocupacional. Pueden identificarse riesgos laborales relacionados globalmente con el trabajo en general, y además algunos riesgos específicos de ciertos medios de producción." (Gaps, A., Casanellas et al, 1981)

Durante el Primer Seminario Latinoamericano de Salud Ocupacional, se afirmó que "la salud ocupacional está ligada a factores económicos, sociales, políticos e históricos, lo cual ha condicionado que en América Latina el desarrollo de estos programas haya tenido características especiales y muy diferentes de los problemas que presentan los países industrializados. La definición general de enfermedad profesional no ha sido todavía alcanzada, pero exige como condición fundamental que pueda ser imputable a causas relacionadas con el trabajo realizado" (1964)

Ya definido estos términos es necesaria una revisión de la situación actual en Ecuador en cuanto a la materia, las necesidades y hacia donde debe apuntar la resolución del problema

El sistema de salud en Ecuador está constituido por las entidades públicas, privadas, autónomas y comunitarias que se articulan funcionalmente sobre la base de principios, políticas, objetivos y normas comunes con la finalidad mejorar el nivel de salud y vida de la población ecuatoriana y hacer efectivo el ejercicio del derecho a la salud. 
El Ministerio de Salud Pública da estas cifras de su capacidad de aporte: instalada 1863 unidades operativas (169 Áreas de Salud, 127 hospitales, 152 centros de salud, 1127 subcentros de salud, 434 puestos de salud), 8942 camas y 27.633 personal operativo. (2009)

Según proyecciones del Instituto Nacional de Estadística y Censos (INEC), Ecuador cuenta con 14.2 millones de habitantes, $50.1 \%$ son hombres y $49.9 \%$ mujeres. Alrededor de $66 \%$ de la población total del país es urbana y 33\% reside en las cinco ciudades más importantes del país. Sin embargo, la cantidad de centros de salud tanto de la red pública como privada, se hacen insuficientes... (2003)

Con estos datos aportados de diversas fuentes es notoria la insuficiencia en materia de salud tanto en centros como en atención primaria y en consecuencia el aumento en los riesgos de contaminación se hace más alarmantes, fundamentalmente los biológicos y los que pueden ocasionar accidentes.

Sobre todo en el sistema de salud pública, la vulnerabilidad de los trabajadores se hace mayor, en tanto las medidas de protección son menores y son menores porque no termina de establecerse una política de Estado hacia los centros de salud pública y la prevención de enfermedades de los trabajadores de este sistema.

Existe un precedente en Ecuador sobre la materia de seguridad y salud ocupacional, desde el Estado se toma la decisión de que la Dirección de Control y Mejoramiento de la Salud Pública, a través del Liderazgo de Salud Ambiental del Ministerio de Salud Pública, con el apoyo de la Organización Panamericana de la Salud, diseñe y ejecute el Proyecto de Hospitales Saludables, destinado al fortalecimiento de la gestión de la salud y seguridad ocupacional en 3 hospitales de Ecuador 
Los efectos resultaron positivos y desde allí nace el planteamiento de que deben extenderse a los demás hospitales del país, ante la necesidad de mitigar los riesgos a los que están expuestos los trabajadores y en concordancia con las políticas institucionales de protección de la salud, la seguridad y el bienestar de los mismos,

Según este proyecto el funcionamiento de los hospitales debiera ya contar con las condiciones óptimas de saneamiento, iluminación, conformación arquitectónica, por no mencionar la dotación de insumos y materiales necesarios para realizar las actividades por parte del personal.

Sin embargo, la verdad de los hospitales y centros de atención en el sector público en el Ecuador, es que reina la carencia de todo tipo de materiales e insumos, así como el diseño arquitectónico, obedece en la mayoría de las ocasiones a paradigmas pasados que resultan inadecuados para la atención de pacientes.

“La Seguridad y Salud Ocupacional (SySO) es una multidisciplina en asuntos de protección, seguridad, salud y bienestar de las personas involucradas en el trabajo. Los programas de seguridad e higiene industrial buscan fomentar un ambiente de trabajo seguro y saludable. El SySO también incluye protección a los compañeros de trabajo, familiares, empleadores, clientes, y otros que podan ser afectados por el ambiente de trabajo.” (Apaza, 2012)

\section{Importancia del problema}

El Ministerio de la salud, frente al reto de organizar el Sistema Nacional de Salud y el Subsistema Nacional de Salud y Seguridad de los Trabajadores, conforme los establece el Instrumento Andino de Seguridad y Salud, y de fortalecer la Rectoría de la Autoridad Sanitaria, pone en marcha los resultados obtenidos durante el año 2007 mediante la ejecución del proyecto de

\footnotetext{
62 Vol. 4, núm. 4, octubre 2018, pp. 57-68 
Hospitales Saludables y que impulsaron precisamente la organización de los Servicios Operativos que hacen factible el cumplimiento de ese importante reto, inmerso en la política gubernamental de protección integral de la salud, la seguridad y el bienestar de los trabajadores en general y particularmente de los trabajadores sanitarios. Es en esa línea que este Ministerio promueve la diseminación de la sistematización acopiada durante la ejecución del Proyecto de Hospitales Saludables y que se traduce en la estructuración del presente Manual, que a no dudarlo servirá de base para el desarrollo del sistema de gestión de la Salud y Seguridad Ocupacional, a favor de los trabajadores sanitarios del país, en acatamiento de las normas nacionales e internacionales que rigen sobre este ámbito de la gestión ministerial. De manera inmediata se extenderán los beneficios a los establecimientos de salud del MSP, de las principales ciudades y paulatinamente se irán estructurando en todos los lugares en donde existen establecimientos de salud pública. En el mediano y largo plazo se extenderán estas normas a todos los establecimientos de salud del país, tanto públicos como privados.

Las autoridades de los establecimientos de salud son los encargados implementar y hacer cumplir los contenidos y alcances del presente Manual, en estrecha y directa colaboración con la Unidad de Salud Ambiental del Nivel Central, que se encargará de realizar el seguimiento y la evaluación pertinentes y de elevar a conocimiento de las instancias superiores el curso de tales acciones. El Ministerio a mi cargo exhorta a todos los empleados y trabajadores para que se cumplan la Ley Orgánica de Salud, a través del cumplimiento del presente Manual, por cuanto guardan entre sí evidente coherencia, de igual forma con las demás normativas nacionales e internacionales relacionadas con la salud y seguridad en el trabajo, principalmente con las recomendaciones de la OMS, de la OIT y del Instrumento Andino de Seguridad y Salud en el Trabajo de la CAN, normativas que se basan precisamente en la legislación y en la práctica nacional. Finalmente, este 
Ministerio hace propicia la ocasión para agradecer la cooperación de la Organización Panamericana de la Salud, Representación en el Ecuador y Washington, para el desarrollo del Proyecto Hospitales Saludables y la elaboración del presente Manual, así como la participación de todos y cada uno de los Hospitales involucrados en el Proyecto, sus Comités de Salud y Seguridad y más Servicios Operativos, destacando la labor cumplida por el Liderazgo de Salud Ambiental para el desarrollo y fortalecimiento de la Gestión de la Salud y Seguridad en el país.

\section{Metodología.}

Se realizará una revisión crítica de diversas fuentes bibliográficas citadas y las consultadas, para conocer y reconocer conceptos como seguridad laboral, salud ocupacional, riesgo laboral.

El tema se hace imprescindible estudiarlo en este momento porque las dinámicas de relación laboral y social han venido cambiando, esto permite incorporar actualizaciones, por ejemplo la salud mental como factor a ser estudiado como factor dentro de lo que se considera salud ocupacional y dentro del riesgo laboral.

El tema es visto desde varios enfoques por lo tanto es necesario converger en la aplicación de varios métodos teóricos y empíricos. La observación, el análisis y la síntesis, la inducción y la deducción y el análisis histórico-lógico, estudios comparativos, convergieron como la estrategia metodológica.

Toda esta estrategia, deriva en la observación de los datos y el análisis de resultados que permiten obtener conclusiones útiles para el análisis y cambios en los centros de salud ecuatorianos. 
Los estudios comparativos de tipo observacional se harán a partir de fuentes de información secundaria; normativa legal ecuatoriana vigente aplicable sobre Seguridad y Salud Ocupacional y, la Norma ISO 26000 (Guía de Responsabilidad Social) en su apartado 6.4.6.2. Se ha elegido esta norma por estar reconocida a nivel internacional y, Ecuador fue parte integrante en su elaboración.

\section{Resultados.}

La Seguridad y Salud Ocupacional es una instancia que se ha instaurado en los procesos laborales y que se preocupa por las cosas que les puede afectar a los trabajadores. En este sentido, es importante establecer programas y medidas de prevención para concientizar sobre esta problemática en base a estadísticas confiables

No se debe plantear que la salud de los empleados es un medio para alcanzar una buena imagen reputacional, la finalidad es lograr un ambiente de trabajo saludable y que éste se convierta en un valor estratégico de la compañía. Las consecuencias de una mala política suponen un coste social y económico importante, debido a un menor desempeño, absentismo, rotación, etc., debido fundamentalmente a la insatisfacción laboral.

Según María Martínez (2017) la participación de los trabajadores es fundamental en materia de seguridad y salud para lo cual las organizaciones que cuenten con más de quince trabajadores deben organizar un organismo paritario denominado Comité y/o Sub Comités de Seguridad y Salud conformado por representantes de los trabajadores y del empleador el cual se encarga entre otras funciones, de promover la observancia de las disposiciones sobre prevención de riesgos profesionales.

\footnotetext{
65 Vol. 4, núm. 4, octubre 2018, pp. 57-68 Karen J. Cedeño-Álava; María Elena De la Cruz Santillán; María J. Zambrano-Zambrano; Gema J. Cantos-Alcívar; Stalin A. Intriago-Miranda; Rina G. Soledispa-Canizares
} 
Es por ello, en Ecuador es de cumplimiento obligatorio el gestionar la seguridad y salud laboral por parte de la alta dirección en donde es clave su compromiso; entre otras cuestiones, el formular la Política de Seguridad y Salud pues se marca un objetivo empresarial en esta materia garantizando recursos y, manifestando el compromiso de cumplir con la legislación técnico legal vigente en el país y el de garantizar las óptimas condiciones de trabajo.

\section{Conclusión.}

Para concluir el tema y después de las observaciones y lecturas.se proponen algunos principios como aporte al tema de la seguridad y el riesgo laboral

La elección de los equipos de trabajo de las sustancias y de los métodos de trabajo y de producción se efectuará de manera que se reduzcan los efectos negativos para la salud y se atenúan e se efectuará de manera que se reduzcan los efectos negativos para la salud y se atenúan el trabajo monótono y repetitivo. Para ello se tendrá en cuenta, en todo momento, la evolución de la técnica.

La prevención de riesgos laborales se planificará logrando un conjunto coherente que integre la técnica, la organización, las relaciones sociales, la influencia de los factores ambientales y todo lo relativo a las condiciones de trabajo.

Las medidas de protección colectiva serán prioritarias frente a los sistemas de protección individual

Se tendrán en cuenta las capacidades profesionales de los trabajadores en materia de seguridad y salud en el momento de encomendarles las tareas, dando las debidas instrucciones y 
asegurando que sólo los que hayan recibido formación suficiente y adecuada puedan acceder a los lugares en los que puedan existir o generarse peligros

A la hora de tomar medidas preventivas se tendrá en cuenta la evolución de la técnica, con el fin de procurar disponer de los sistemas de protección más idóneos en cada circunstancia. Se adecuarán los aspectos materiales a las condiciones de cada trabajador y se preverán las distracciones o imprudencias no temerarias que se pudieran cometer.

La prevención de riesgos laborales es un requisito básico para el logro de la calidad. Un trabajo bien hecho sólo se considerará como tal cuando esté hecho en las debidas condiciones de seguridad.

Se deberá promover en todos los ámbitos del trabajo en equipo y la cooperación entre los diversos estamentos de la empresa. También es básico colaborar con los clientes y proveedores para mejorar continuamente la manera de fabricar, transportar y utilizar los productos.

Las personas constituyen el valor más importante que garantiza nuestro futuro.

La seguridad y la salud de los trabajadores será uno de los objetivos permanentes y fundamentales, de la misma forma que lo son la calidad, la productividad y la rentabilidad de las actividades.

Los accidentes de trabajo o cualquier lesión generada en el mismo, son fundamentalmente fallos de verificables y por tanto se debe adoptar las medidas para la identificación, evaluación y control de los posibles riesgos.

\footnotetext{
67 Vol. 4, núm. 4, octubre 2018, pp. 57-68 


\section{Bibliografía}

Ministerio de Salud Publica. (2009). Manual de Procedimientos para la Gestion de los Comites de Salud y Seguridad Ocupacional en Establecimientos de Salud.

Apaza, R. (28 de 12 de 2012). Seguridad y Salud Ocupacional: Definicion. Obtenido de https://www.rubenapaza.com/2012/12/seguridad-y-salud-ocupacional-definicion.html

Gaps, A., Casanellas et al. (1981). El medi ambient i la salut. Colegio Médico de Barcelona: Colección Salud y Sanidad 4.

Martinez, M. (2017). Seguridad y Salud Ocupacional en Ecuador: Contribución Normativa a la Responsabilidad Social Organizacional. INNOVA Research Journal, 58-68.

Organización Panamericana de la Salud. (1964). Primer Seminario Latinoamericano de Salud Ocupacional. São Paulo, Brasil.

Ortiz , P., \& Albán , E. (2003). Ecuador: estimaciones y proyecciones de población 1950-2025. Quito: Instituto Nacional de Estadística y Censos. 